\title{
Current status of thrombolytic agents in acute cerebrovascular disease
}

\author{
ANTHONY P. FLETCHER \\ Washington University School of Medicine, St Louis, Missouri
}

Current therapy of the acute cerebrovascular attack is essentially directed to preservation of life during the acute phases and to amelioration of the functional effects of neurological deficit in the later phase. Thus, there is an urgent need for investigation of methods capable of increasing blood supply to ischaemic brain tissue capable of salvage but potentially early enough to reduce the effects of hypoxia. Moreover, such treatment, if applied during the stage of progressive stroke (or stroke-in-evolution), would prove effective in halting lesion progression, in the salvaging of ischaemic brain areas, and possibly in preventing later recurrence of this disorder.

Medical treatment with thrombolytic (clotlysing) agents would appear to possess the following advantages:

1 Unlike surgical therapy, severely restricted in scope by technical considerations of anatomical access, thrombolytic therapy is applicable to the treatment of both intracranial and extracranial arterial vascular lesions and also to the increasingly recognized syndromes resulting from acute thrombotic involvement of the cerebral venous sinuses.

2 Thrombolytic therapy may be employed in cases where multiple vascular occlusive lesions exist, and its use in the pure therapeutic situation would require only that a firm pathological diagnosis-as apart from an additional precise anatomical diagnosis-be established. Even after extensive angiographic study, which carries some hazard, the site of vascular occlusion is not always evident in patients with cerebral thrombosis or embolism. Thus, the lessened requirement for precise anatomical lesion localization when using thrombolytic therapy would be of great practical advantage and substantially extend the utility and applicability of the treatment.

3 The factors governing the recovery of ischaemic but salvagable brain areas are incompletely understood, but if, as is suspected, restoration of areas of microthrombosis, thrombolytic agents might produce clinical benefit even if the apparent causative thrombus were not itself lysed. Furthermore, the treatment might be reasonably expected to prevent potentially significant secondary thrombosis and possibly guard against future recurrence.

4 It may reasonably be postulated, in view of the biochemical data establishing the alteration of plasma fibrinogen $D_{20 \text {,w }}$ to 2.5 or higher during urokinase therapy, that blood rheological properties, especially at low shear rates, will be substantially altered. This drug effect may be of unusual importance in acute cerebrovascular disease, for it has recently been demonstrated in the author's laboratory that fibrinogen molecular distribution patterns are usually grossly disturbed and blood rheology correspondingly altered. Thus, it may be that early rapid improvement observed in some treated patients is related to alterations in blood rheological behaviour.

5 Since thrombolytic therapy is specific against fibrin and fibrin-containing thrombi and emboli, lysis of the vascular obstruction may be accomplished without further damage to the arterial wall, an important consideration in preventing recurrent embolism.

On the other hand, there are potential hazards to thrombolytic therapy in acute cerebral thrombosis or embolism. The descriptive term 'potential hazards' is used since there is a lack of reliable pathophysiological information in areas relevant to quantification or risk. For instance, the relationship between 'red' and 'white' infarction is unclear, and the factors causing the development of red infarction in one patient and white in another can only be conjectured. Consequently, the important question as to whether thrombolytic therapy, administered in such a manner as to produce only minor disturbance of coagulation function, does or does not favour the conversion of white to red infarction, cannot be answered except by experience. Similarly, in the unfortunate event that cerebral thrombosis was incorrectly diagnosed in a patient suffering from cerebral haemorrhage, there is reason to believe that thrombolytic therapy would be deleterious, yet at the present time, no realistic estimate of hazard is possible.

Only limited experience with thrombolytic agents 
in cerebrovascular disease is presently available. Of the two statistically controlled therapeutic trials that have been published, the first with a plasmin/ streptokinase mixture (Thrombolysin) suggested that the treatment was without value, while the second trial of streptokinase plus anticoagulants versus a control group of anticoagulant-treated patients suggested that though lysis of the causative thrombus occurred more frequently in the treated than in the control group, judged by mortality and morbidity criteria, the treated group responded less well to therapy than did the control group.

However, for various well known reasons, streptokinase is a far from ideal drug for use in acute cerebrovascular disease and serious exploration of the potential of thrombolytic therapy in this area required the availability of a superior thrombolytic agent such as urokinase.

Urokinase therapy does not appear to present any special difficulty or hazard in the patient with acute cerebrovascular disease. Urokinase dosage requirements, calculated on a patient body-weight basis, are similar to those of patients suffering from other thromboembolic disease states. High levels of plasma thrombolytic activity are induced by the therapy with only minimal disturbance to blood coagulation function.

Clinically, no unusual haemorrhagic phenomena were observed except that, similarly to other patients treated with urokinase, haemorrhage sometimes occurred at surgical incision sites or at sites of recent arterial catheter insertion.

While no valid assessment of urokinase therapeutic effect can be made with this small number of stroke patients, it should be mentioned that there was only one death (not attributable to the treatment). Deterioration of patients during the time of treatment infusion was not observed, and, five patients suffering from severe venous sinus thrombosis recovered following therapy, in three instances, with apparently unusual rapidity and completeness.

These findings suggest that a larger trial of urokinase therapy in acute cerebrovascular disease is certainly feasible and should be undertaken.

\title{
Observations of enzyme elevations in the serum during streptokinase treatment
}

\author{
E. SCHMIDT, H. POLIWODA, V. BUHL, K. AlEXANDER, AND \\ F. W. SC HMIDT
}

From Medizinische Hochschule, Hannover, Germany

Side effects of fibrinolytic therapy with streptokinase are well known. Casual observations of enzyme elevations in the serum of patients with chronic arterial obstruction of the lower extremities during streptokinase treatment (Martin, Schoop, and Zeitler, 1969) suggested that a planned study of 24 unselected patients should be performed (Schmidt, Poliwoda, Buhl, Alexander, and Schmidt, 1969).

The enzymes were chosen with regard to their value in differentiating distinct cellular lesions: the amino transferases (GOT and GPT), together indicating disturbed permeability of liver cells, the mitochondrial glutamate dehydrogenase (GLDH) as an index of severe cellular damage, alkaline phosphatase (SAP) and $\gamma$-glutamyl transpeptidase (GGTP) for the evaluation of cholestasis and cholinesterase (CHE) as a measure of the capacity of $D$ protein synthesis in the liver. Lactic dehydrogenase (LDH) and creatinephosphate kinase (CPK) were added to the profile as indices of a possible involvement of red blood cells and skeletal muscle. Enzyme determinations were done at least twice before therapy in order to establish reliable initial values. With few exceptions the activities of all enzymes were within the normal range. During streptokinase treatment determinations of enzyme activities were : done at 12-hour intervals from the first to the fifth day, daily up to the eighth day, and after this, at varying intervals up to three months.

The response of our patients to fibrinolytic $\mathbb{D}$ therapy as reflected by their enzyme patterns in serum can be divided into three different stages. 